\title{
Anemia do atleta (II): incidência e conduta terapêutica
}

\author{
Ramón José Nuviala Mateo ${ }^{1}$ e María Gloria Lapieza Laínez ${ }^{2}$
}

\section{INTRODUÇÃO}

Os estados deficitários de ferro $(\mathrm{Fe})$ não são exclusivos das mulheres atletas, embora nas mulheres a sua incidência seja muito menor. Duas razões importantes explicam essa diferença: por um lado a maior ingesta energética dos homens e por consequiência de Fe; e por outro lado as suas menores necessidades desse micronutriente.

\section{O FERRO E A MULHER ATLETA}

Conforme já comentado, a causa mais freqüente desse precário equilíbrio do $\mathrm{Fe}$ na mulher é uma ingesta inadequada. Assim, Clement e Asmundson ${ }^{37}$ encontraram uma elevada prevalência de ingestão inadequada de $\mathrm{Fe}$ nas mulheres atletas $(90,9 \%)$ em relação aos atletas do sexo masculino $(2,6 \%)$. Por outro lado, isto se traduz em baixos níveis de ferritina sérica nas atletas em relação aos dos atletas homens ${ }^{57}$. Esta segunda parte se concentrará nos estados deficitários de Fe na mulher atleta.

A maioria dos autores encontrou ingestas de Fe total nas mulheres abaixo das recomendadas como mínimas pelas $\mathrm{RDA}$, conforme pode ser visto na tabela 1 . Nuviala et al. ${ }^{79}$ em jogadoras de basquete, Haymes e Spillman ${ }^{84}$ em velocistas e Steinbaugh ${ }^{85}$ em nadadoras e bailarinas referem ingestas acima dos $15 \mathrm{mg}$ por dia.

Na tabela 1, também estão demonstrados os percentuais de mulheres com ingestão insuficiente de $\mathrm{Fe}$, valores referidos somente por alguns dos autores. Esses percentuais

1. Serviço de Bioquímica Clínica, Hospital Clínico Universitário de Zaragoza.

2. Departamento de Farmacologia e Fisiologia, Faculdade de Medicina de Zaragoza, Zaragoza, Espanha.

Endereço para correspondência:

Dr. Ramón J. Nuviala

Servicio de Bioquímica Clínica

Hospital Clínico Universitario

Avda. San Juan Bosco, 15

50009 - Zaragoza - Espanha

E-mail: glapieza@posta.unizar.es

\begin{tabular}{|c|c|c|c|}
\hline \multicolumn{4}{|c|}{$\begin{array}{c}\text { TABELA } 1 \\
\text { Ingestão de Fe total e percentual de } \\
\text { mulheres atletas com déficit de ingesta }\end{array}$} \\
\hline Autor & Modalidade & $\begin{array}{c}\text { Fe } \\
\text { mg/dia }\end{array}$ & $\begin{array}{l}\text { Mulheres } \\
\text { com déficit }\end{array}$ \\
\hline Reggiani et al. ${ }^{71}$ & Ginástica & 6,2 & - \\
\hline Nowak et al. ${ }^{72}$ & Basquete & 10,0 & - \\
\hline Brown et al. ${ }^{73}$ & Atletismo & 10,6 & - \\
\hline Diehl et al. ${ }^{74}$ & Hóquei & 11,0 & - \\
\hline Perron e Endres ${ }^{75}$ & Vôlei & 11,0 & $69,0 \%$ \\
\hline Pate et al. ${ }^{76}$ & Corrida & 11,2 & - \\
\hline Moffat ${ }^{38}$ & Ginástica & 11,2 & $100,0 \%$ \\
\hline Watkin et al. ${ }^{77}$ & Maratona & 11,7 & - \\
\hline Erp-Baart et al. ${ }^{78}$ & Handebol & 12,0 & - \\
\hline Clement e Asmundson ${ }^{37}$ & Corrida & 12,5 & $90,9 \%$ \\
\hline Nuviala et al.79 & $\begin{array}{l}\text { Caratê } \\
\text { Handebol } \\
\text { Corrida } \\
\text { Basquete }\end{array}$ & $\begin{array}{l}12,7 \\
12,9 \\
13,6 \\
15,8\end{array}$ & $\begin{array}{l}63,1 \% \\
80,0 \% \\
68,0 \% \\
40,0 \%\end{array}$ \\
\hline Faber e Benadé 80 & Atletismo & 13,3 & $70,0 \%$ \\
\hline Deuster et al. ${ }^{81}$ & Corrida & 13,9 & $76,5 \%$ \\
\hline Lukasky et al. ${ }^{82}$ & Natação & 13,8 & - \\
\hline Newhouse et al. ${ }^{83}$ & $\begin{array}{l}\text { Atividade } \\
\text { física } \\
\text { recreativa }\end{array}$ & 14,6 & - \\
\hline Haymes e Spillman ${ }^{84}$ & $\begin{array}{l}\text { Fundistas } \\
\text { Velocistas }\end{array}$ & $\begin{array}{l}14,9 \\
15,0\end{array}$ & $\begin{array}{l}36,0 \% \\
33,0 \%\end{array}$ \\
\hline Steinbaugh 85 & $\begin{array}{l}\text { Basquete } \\
\text { Natação } \\
\text { Balé }\end{array}$ & $\begin{array}{l}14,0 \\
16,0 \\
19,0\end{array}$ & $\begin{array}{l}- \\
- \\
-\end{array}$ \\
\hline
\end{tabular}

Traduzido, com permissão por escrito, do original: Mateo RJN, Laínez MGL. La anemia del deportista (II). Incidencia y pautas terapeuticas. Arch Med Deporte 2000;75:47-57. 
variam entre os $33 \%$ de Haymes e Spillman ${ }^{84}$ em velocistas até um máximo de $100 \%$ relatado por Moffat ${ }^{38} \mathrm{em}$ ginastas, valor que parece superestimado, já que levou em conta a ingesta mínima de Fe de $18 \mathrm{mg}$ ao dia, segundo as RDA anteriores ${ }^{86}$.

Quanto ao percentual da ingestão média de Fe total em relação à RDA, vários autores colocam diferentes valores. Desta forma, Rowland e Kelleher ${ }^{87}$ relatam que as suas nadadoras chegam somente a $43 \%$ destas; Perron e Endres ${ }^{75}$ em praticantes de atletismo chegam a $60,0 \%$; Moffat ${ }^{38}$ relata $62,5 \%$ e Watkin et al. ${ }^{77}$ e Lampe et al. ${ }^{88}$ em maratonistas relatam $65,0 \%$ e $78,0 \%$ respectivamente, levando em conta que todos esses autores estabelecem o mínimo recomendado em $18 \mathrm{mg}$ ao dia ${ }^{86}$. Por último, e considerando as necessidades atuais de $15 \mathrm{mg}$ por dia, Nutter ${ }^{89}$ em vários grupos de desportistas estabeleceu que o consumo de $\mathrm{Fe}$ foi abaixo de $70 \%$ das RDA.

Ao passo que os trabalhos que avaliam a ingestão de $\mathrm{Fe}$ total são numerosos, os que analisam a ingestão de Fe hemo nas atletas são bem mais escassos. Considerando que a ingestão mínima de Fe total na mulher deve ser de $15 \mathrm{mg}$ ao dia e que desse total pelo menos $10 \%$ deve ser de procedência hemínica ${ }^{27}$, deduz-se que a ingestão de Fe hemo ou hemínico deve ser em torno de $1,5 \mathrm{mg}$ ao dia.

Entre os diferentes autores (tabela 2), observa-se que tanto Haymes e Spillman ${ }^{84}$, quanto Weight et al. ${ }^{90}$ e Snyder et al. ${ }^{40}$ em corredoras, e Nuviala et al..$^{79}$ em caratecas não conseguem observar um mínimo de $1,5 \mathrm{mg}$ ao dia; enquanto que Nuviala et al..$^{79} \mathrm{em}$ corredoras e jogadoras de handebol e basquete da mesma forma que Erp-Baart et $a l .{ }^{78}$ em corredoras, nadadoras, ginastas e jogadoras de vôlei e basquete referem ingestas de $\mathrm{Fe}$ hemo que ultrapassam os valores mínimos recomendados.

A dosagem da ferritina sérica como parâmetro indicador de um estado deficitário de $\mathrm{Fe}$ é fundamental em um controle adequado do metabolismo desse mineral, sendo muitos os trabalhos que analisam a mulher atleta. $\mathrm{Na}$ tabela 3 estão ilustrados os valores médios de ferritina encontrados por diferentes autores para dife-

TABELA 2

Ingestão de Fe hemo em mulheres atletas

\begin{tabular}{|c|c|c|}
\hline Autor & Modalidade & $\begin{array}{c}\text { Fe hemo } \\
\text { (mg ao dia) }\end{array}$ \\
\hline Haymes e Spillman ${ }^{84}$ & $\begin{array}{l}\text { Fundistas } \\
\text { Velocistas }\end{array}$ & $\begin{array}{l}0,90 \\
1,50\end{array}$ \\
\hline Weight et al.90 & Corrida & 1,06 \\
\hline Snyder et al.40 & Corrida & 1,20 \\
\hline Nuviala et al. ${ }^{79}$ & $\begin{array}{l}\text { Caratê } \\
\text { Corrida } \\
\text { Handebol } \\
\text { Basquete }\end{array}$ & $\begin{array}{l}1,45 \\
1,53 \\
1,75 \\
2,15\end{array}$ \\
\hline Erp-Baart et al. ${ }^{78}$ & $\begin{array}{l}\text { Corrida } \\
\text { Natação } \\
\text { Ginástica } \\
\text { Vôlei } \\
\text { Basquete }\end{array}$ & $\begin{array}{l}1,60 \\
1,90 \\
2,30 \\
2,50 \\
3,20\end{array}$ \\
\hline
\end{tabular}

TABELA 3

Valores médios de ferritina sérica em mulheres atletas e prevalência do déficit de Fe

\begin{tabular}{lllll}
\hline Autor & Modalidade & Ferritina & $\%$ & Limite
\end{tabular}

\begin{tabular}{|c|c|c|c|c|}
\hline Haymes e Spillman ${ }^{84}$ & Corrida & 15,8 & 31,2 & 12 \\
\hline Brown et al. ${ }^{73}$ & Atletismo & 16,8 & 44,0 & 12 \\
\hline Colt e Heyman ${ }^{91}$ & Corrida & 17,8 & 28,1 & 12 \\
\hline Rowland e Kelleher 87 & Natação & 18,9 & 46,7 & 12 \\
\hline Snyder et al. ${ }^{40}$ & Corrida & 19,8 & 22,2 & 12 \\
\hline Durstine et al..$^{92}$ & Corrida & 34,0 & 31,2 & 12 \\
\hline López et al.39 & $\begin{array}{l}\text { Ginástica } \\
\text { Natação }\end{array}$ & $\begin{array}{l}17,6 \\
19,6\end{array}$ & $\begin{array}{l}80,0 \\
55,5\end{array}$ & $\begin{array}{l}20 \\
20\end{array}$ \\
\hline Nickerson e Tripp ${ }^{93}$ & Corrida & - & 61,1 & 20 \\
\hline Nuviala et al. ${ }^{79}$ & $\begin{array}{l}\text { Caratê } \\
\text { Handebol } \\
\text { Basquete } \\
\text { Corrida }\end{array}$ & $\begin{array}{l}26,7 \\
26,7 \\
28,1 \\
34,6\end{array}$ & $\begin{array}{l}47,3 \\
35,0 \\
45,0 \\
28,0\end{array}$ & $\begin{array}{l}20 \\
20 \\
20 \\
20\end{array}$ \\
\hline Newhouse et al.83 & $\begin{array}{l}\text { Atividade física } \\
\text { recreativa }\end{array}$ & 28,8 & 38,7 & 20 \\
\hline Lampe et al. ${ }^{88}$ & Maratona & 30,0 & 22,2 & 20 \\
\hline Weight et al. ${ }^{94}$ & $\begin{array}{l}\text { Balé } \\
\text { Corrida }\end{array}$ & $\begin{array}{l}38,5 \\
45,2\end{array}$ & $\begin{array}{l}26,7 \\
16,7\end{array}$ & $\begin{array}{l}20 \\
20\end{array}$ \\
\hline Clement e Asmundson ${ }^{37}$ & Corrida & 27,9 & 81,8 & 25 \\
\hline
\end{tabular}


rentes modalidades desportivas. As cifras médias descritas são muito variáveis oscilando entre valores mais baixos de $15,8 \mathrm{ng} \cdot \mathrm{ml}^{-1}$ de Haymes e Spillman ${ }^{84}$ em corredoras e de $16,8 \mathrm{ng} \cdot \mathrm{ml}^{-1}$ de Brown et al. ${ }^{73}$ em praticantes de atletismo, até chegar a um valor de $45,2 \mathrm{ng} \cdot \mathrm{ml}^{-1}$ relatado por Weight et al. ${ }^{94}$ em corredoras.

De acordo com os diferentes estudos revisados, existe uma disparidade de critérios quanto à concentração mínima de ferritina sérica considerada como indicadora de um estado deficitário de $\mathrm{Fe}$. Alguns trabalhos consideram $12 \mathrm{ng} \cdot \mathrm{ml}^{-1}$ enquanto que outros consideram $20 \mathrm{ng} \cdot \mathrm{ml}^{-1}$. Na atualidade, parece que este último valor é mais aceito e desta forma Wick et al. ${ }^{62}$ estabelecem uma clara diferença entre os $20 \mathrm{ng} \cdot \mathrm{ml}^{-1}$ que consideram como limite da deficiência de Fe de depósito e os $12 \mathrm{ng} \cdot \mathrm{ml}^{-1}$ que indicam uma deficiência do transporte de Fe. Por outro lado, comprovou-se que com níveis de ferritina sérica inferiores a 20ng $\cdot \mathrm{ml}^{-1}$ há ausência de Fe nos macrófagos da medula óssea ${ }^{61}$.

Existem ainda uma série de circunstâncias que podem modificar os níveis séricos de ferritina e que devem ser lembrados na hora de interpretar os resultados. Entre estas podemos citar:

a) Os níveis de ferritina sérica variam em proporção direta com as reservas orgânicas totais de $\mathrm{Fe}$, de tal forma que $1 \mathrm{ng} \cdot \mathrm{ml}^{-1}$ de ferritina sérica corresponde a $8-10 \mathrm{mg}$ de Fe de depósito ${ }^{58}$. Entretanto, deve-se levar em conta que a ferritina é uma proteína que se comporta como um reagente de fase aguda positivo, aumentando em resposta a um processo inflamatório ${ }^{95}$. Nos casos em que se detectem níveis muito elevados de ferritina sérica ou quando existirem quadros de anemias manifestas com valores normais de ferritina, deve-se considerar a possibilidade de determinar conjuntamente outros reagentes de fase aguda positivos tais como a proteína $C$ reativa, a ceruloplasmina, $\alpha_{1-}$ antitripsina, $\alpha_{1}$-glicoproteína ácida, $\alpha_{2}$-macroglobulina e outros marcadores inflamatórios, com o objetivo de descartar processos inflamatórios ou infecciosos do organis$\mathrm{mo}^{96}$.

b) Não devem ser realizadas dosagens de ferritina sérica após atividade física de grande intensidade já que os seus níveis sofrem importantes aumentos nessas circunstâncias ${ }^{45}$, podendo demorar vários dias para normalizarem; não refletirão assim de modo exato as reservas corporais de $\mathrm{Fe}^{88}$.

c) Deve-se perguntar às mulheres atletas sobre o uso de anticoncepcionais já que estes reduzem as perdas sanguíneas menstruais e por outro lado aumentam a absorção intestinal de Fe, resultando em um aumento dos depósitos de $\mathrm{Fe}^{97}$. Um claro exemplo disso são os níveis séricos significativamente maiores de ferritina encontrados em atletas que tomam anticoncepcionais em relação às que não os tomam $^{83}$.

d) Por último, deve-se levar em conta que as concentrações de ferritina sérica relatadas por alguns autores podem estar parcialmente distorcidas, devido à administração de suplementos farmacológicos e ao seu conseqüente aumento.

Na tabela 3 também estão demonstrados os percentuais de mulheres com déficit de Fe, estabelecendo-se claramente as diferenças existentes quanto ao limite de ferritina proposto. Está também demonstrado o limite de $25 \mathrm{ng} \cdot \mathrm{ml}^{-1}$ proposto por Clement e Asmundson ${ }^{37}$ para as suas corredoras, entre as quais é relatado um percentual de $81,8 \%$ de casos de déficit.

Quanto à prevalência de anemia entre as atletas e a sua maior frequiência em relação às mulheres não ativas, existe uma ampla disparidade de opiniões. Assim, Pate ${ }^{11}$, Seiler et al. ${ }^{48}$, Haymes e Spillman ${ }^{84}$, Balaban et al. ${ }^{98}$ e Douglas ${ }^{99}$ relatam ausência ou um baixíssimo percentual de anemias entre os seus atletas. Por outro lado, outros autores descrevem francas anemias entre as mulheres atletas. Na tabela 4 , estão os valores médios de hemoglobina encontrados por diferentes autores em suas atletas assim como o percentual de anemias entre elas, com valores que variam entre $0 \%$ encontrado por Nuviala et al. ${ }^{79} \mathrm{em}$ corredoras e $21,4 \%$ encontrado por Weight et al..$^{94}$ em bailarinas.

Em outras ocasiões e embora pareça paradoxal, foram descritas concentrações mais elevadas de hemoglobina nas atletas do que nas mulheres sedentárias; desta forma, Balaban et al. ${ }^{98}$ relatam valores médios de $12,8 \mathrm{~g} \cdot \mathrm{dl}^{-1}$ no gru-

\begin{tabular}{|c|c|c|c|}
\hline \multicolumn{4}{|c|}{$\begin{array}{c}\text { TABELA } 4 \\
\text { Valores médios de hemoglobina e } \\
\text { prevalência de anemia em atletas mulheres }\end{array}$} \\
\hline Autor & Modalidade & $H b\left(g \cdot d l^{-1}\right)$ & $\begin{array}{c}\text { Prevalência } \\
\text { de anemia (\%) }\end{array}$ \\
\hline Brown et al. ${ }^{73}$ & Atletismo & 12,5 & 12,5 \\
\hline Nuviala et al..$^{79}$ & $\begin{array}{l}\text { Basquete } \\
\text { Caratê } \\
\text { Handebol } \\
\text { Corrida }\end{array}$ & $\begin{array}{l}12,8 \\
12,9 \\
13,2 \\
13,4\end{array}$ & $\begin{array}{r}20,0 \\
10,5 \\
5,0 \\
0,0\end{array}$ \\
\hline Snyder et al. ${ }^{40}$ & Corrida & 13,1 & 11,1 \\
\hline Weight et al. ${ }^{94}$ & $\begin{array}{l}\text { Balé } \\
\text { Corrida }\end{array}$ & $\begin{array}{l}13,2 \\
13,3\end{array}$ & $\begin{array}{l}21,4 \\
11,7\end{array}$ \\
\hline Newhouse et al.83 & $\begin{array}{l}\text { Atividade } \\
\text { física } \\
\text { recreativa }\end{array}$ & 13,7 & 3,6 \\
\hline Durstine et al..92 & Corrida & 14,0 & 13,1 \\
\hline
\end{tabular}


po controle e um valor significativamente maior, de $13,5 \mathrm{~g} \cdot \mathrm{dl}^{-1}$ nas atletas. Também Pellicia e Nucci ${ }^{100}$ encontraram $14,0 \mathrm{~g} \cdot \mathrm{dl}^{-1} \mathrm{em}$ nadadoras, valor significativamente maior do que os $12,8 \mathrm{~g} \cdot \mathrm{dl}^{-1}$ do seu grupo controle, sendo que os autores justificam os resultados pelo fato de que nos esportes de predomínio aeróbico as reservas de Fe aumentam.

Em qualquer caso, o que mais chama a atenção nesta revisão é que a alta prevalência descrita de ingestas deficitárias de Fe contrasta com a baixa incidência de casos de franca anemia. Assim, uma ingestão de Fe abaixo dos níveis recomendados segundo as RDA indica somente um possível risco de deficiência nutricional e não necessariamente significa que o nível da ingesta seja insuficiente para as necessidades individuais reais da atleta.

\section{O FERRO E OS ATLETAS JOVENS}

Desperta um especial interesse o estudo da deficiência de $\mathrm{Fe}$ em populações consideradas de alto risco, como as crianças e os adolescentes, cuja participação desportiva aumentou nitidamente nos últimos anos.

A necessidade de Fe na dieta é maior durante a infância e a adolescência do que em qualquer outro período da vida. Ao contrário do adulto, cuja absorção de Fe não tem outra finalidade senão a de compensar as perdas habituais, as crianças devem acumular Fe adicional para o seu crescimento. Desta forma, a aceleração do crescimento do adolescente e a sua maturação sexual aumentam marcadamente as necessidades de Fe na dieta para compensar as perdas normais e as necessidades próprias do crescimento e no caso dos jovens atletas as perdas ocasionadas pelo próprio exercício físico ${ }^{23,101}$. Portanto, as crianças e adolescentes com atividade física regular são populações de alto risco para deficiência de Fe.

Existem claras evidências, tanto na criança sedentária como no jovem atleta, de que a deficiência de Fe não somente é uma causa de diminuição da capacidade de trabalho físico mas também repercute sobre a aprendizagem e inclusive sobre a própria saúde da criança, afetando de modo negativo as funções gastrointestinal, neurológica e imunológica ${ }^{102,103}$.

Segundo Dallman ${ }^{23}$, os rapazes têm o seu pico de crescimento entre os 11,7 e os 15,3 anos de idade, aumentando marcadamente as suas necessidades de $\mathrm{Fe}$ absorvido que passam de 1,0 para 2,5mg ao dia. Nas jovens o pico de crescimento oscila entre os 9,7 e os 13,3 anos de idade, aumentando as necessidades médias de Fe absorvido para até $1,5 \mathrm{mg}$ ao dia. Após o período de crescimento acelerado, há uma rápida redução da necessidade de Fe nos rapazes, retornando ao seu valor inicial de $1,0 \mathrm{mg}$ ao dia, en- quanto que nas jovens se estabiliza em torno de $1,3 \mathrm{mg}$ ao dia, devido à necessidade de repor as perdas menstruais, embora se calcule que cerca de $20 \%$ das mulheres necessitem de até $2,0 \mathrm{mg}$ ao dia.

Burman ${ }^{104}$ defende que durante o período que vai dos 12 aos 18 anos pode haver um ganho médio de peso de aproximadamente $30 \mathrm{~kg}$ nos rapazes e um pouco menos nas jovens, o que faria necessária uma absorção média de $0,8 \mathrm{mg}$ ao dia, que seria maior durante o período de estirão do crescimento. De Wijn e Pikaar ${ }^{105}$, após um estudo longitudinal realizado em rapazes holandeses de 9 a 17 anos, descrevem que a depleção de $\mathrm{Fe}$ sem anemia ocorre mais freqüentemente nos rapazes durante a puberdade, enquanto que nas jovens esta depleção é mais freqüente durante o período pós-púbere, aumentando nelas a incidência de anemia de $1 \%$ aos nove anos para $7 \%$ aos 17 anos.

$\mathrm{Na}$ Espanha, em um estudo realizado em uma grande amostra de crianças de Cantabria e tomando como parâmetros a ferritina $\left(<12 \mathrm{ng} \cdot \mathrm{ml}^{-1}\right)$ e o índice de saturação da transferrina $(<16 \%)$, observou-se que entre as crianças de 9 a 11 anos havia $12,9 \%$ com ferropenia, enquanto que entre 12 e 14 anos a incidência aumentava para $21,8 \%$ nos rapazes e $14,8 \%$ nas jovens ${ }^{106}$. Em outro estudo realizado em um grupo de crianças de nove a 15 anos em Reus, observou-se que somente $2,1 \%$ tinham níveis de ferritina inferiores a $12 \mathrm{ng} \cdot \mathrm{ml}^{-1}$ e $19,7 \%$ apresentavam um índice de saturação da transferrina abaixo do normal e somente $1,4 \%$ foram diagnosticados como anemia ferropriva ${ }^{107}$.

Da mesma forma que ocorre com os adultos, uma das causas desencadeantes dos estados deficitários de $\mathrm{Fe}$ mais freqüentes é a ingestão insuficiente desse micronutriente. Em um estudo realizado na Comunidade de Madrid ${ }^{108}$ observou-se que $92 \%$ das meninas de 10 a 13 anos e $88 \%$ das de 13 a 16 anos apresentavam ingestas deficitárias de $\mathrm{Fe}$ em relação ao mínimo estabelecido pelas Recomendações Dietéticas. Da mesma forma, Salas et al. ${ }^{109}$ estabelecem que $75,9 \%$ dos rapazes e $93,2 \%$ das jovens de 13 a 16 anos não alcançam a ingesta mínima recomendada para o $\mathrm{Fe}$.

Rowland et al. ${ }^{110}$ referem que a prevalência de déficit de $\mathrm{Fe}$, avaliada unicamente por uma concentração de ferritina inferior a $12 \mathrm{ng} \cdot \mathrm{ml}^{-1}$, era de $40 \%$ em um grupo de corredoras adolescentes e de $46,7 \%$ em nadadoras com idades similares, com diferenças desprezíveis desses grupos de atletas em relação ao grupo controle. Por outro lado, descreveram-se valores médios de ferritina superiores em ginastas rítmicas e nadadoras adolescentes em relação aos de estudantes sedentárias, embora sem diferenças significativas ${ }^{111}$.

Em atletas adolescentes pré-menárquicas foram encontrados déficits na ingestão de Fe de $88,2 \%$ entre as ginastas rítmicas e $88,4 \%$ entre as nadadoras, enquanto que para as jovens menárquicas foram encontrados valores de $100 \%$ 
para as ginastas rítmicas e $88,8 \%$ para as nadadoras ${ }^{39}$. Nessas mesmas amostras observou-se que os níveis médios de ferritina sérica nas atletas menárquicas são marcadamente inferiores aos das pré-menárquicas, tanto nas ginastas como nas nadadoras e inclusive no grupo controle, o que demonstra claramente a influência que tem a presença da menstruação sobre as reservas corporais de Fe.

Romero $^{112}$, em uma ampla revisão realizada com adolescentes de ambos os sexos, demonstrou uma alta prevalência de ingestas deficitárias de Fe tanto entre os adolescentes com atividade física quanto entre os sedentários. Por outro lado, relata um grande percentual de déficits de ferritina $\left(<20 \mathrm{ng} \cdot \mathrm{ml}^{-1}\right)$ entre os atletas adolescentes e da mesma forma que ocorre com as mulheres atletas uma baixa incidência de francas anemias em relação ao elevado percentual de casos com ingesta deficitária de Fe.

Quanto às modificações causadas pelo treinamento e pela competição sobre as reservas de Fe do organismo em atletas adolescentes, Rowland et al. ${ }^{110}$ descrevem reduções dos níveis de ferritina sérica entre as corredoras ao longo da temporada, não sendo encontradas alterações entre as nadadoras. Em outro estudo realizado em nadadores demonstrou-se um pequeno aumento da ferritina nos nadadores adolescentes após quatro meses de treinamento, enquanto que entre as nadadoras o aumento foi significativo ${ }^{113}$.

\section{SUPLEMENTAÇÃO DE FERRO}

A prevenção e o tratamento dos estados deficitários de Fe nos atletas devem ser contemplados de dois modos diferentes: 1) A suplementação nutricional e 2) A suplementação farmacológica.

\section{1) A suplementação nutricional de Fe:}

É de grande importância o controle da ingesta de Fe total e de $\mathrm{Fe}$ hemo nos atletas, com o fim de comprovar se atingem o mínimo estabelecido pelas RDA para o seu sexo e idade ${ }^{21}$.

Para avaliar a ingesta de Fe é recomendada a utilização das avaliações nutricionais, pelo método de registro ou recordatório de 24 horas, que pode ser acrescido de mais dias; ou pelo método da pesagem dos alimentos. Ambos estão sujeitos a certos erros, embora o último seja mais confiável, recomendando-se que seja efetuado ao longo de sete dias, incluindo um fim de semana.

Quando se observa uma ingesta insuficiente de Fe, devese recomendar o consumo de alimentos ricos neste micronutriente, sejam de origem animal (chouriço, mariscos, ostras, mexilhões, fígado, escargôs, carnes vermelhas, etc.) ou de origem vegetal (cereais integrais, germe de trigo, soja em grão, pistache, soja, verduras de folhas escuras, etc.), com maior ou menor conteúdo de Fe de acordo com as tabelas de alimentos publicadas por Mataix et al. ${ }^{114}$. Entretanto, em todas as tabelas de composição de alimentos figura o conteúdo de $\mathrm{Fe}$ dos diferentes alimentos, expresso habitualmente por $100 \mathrm{~g}$ de alimento comestível.

Outro assunto interessante quando se fala da absorção do Fe na dieta diz respeito a possíveis influências com outros nutrientes da dieta, havendo os fatores favorecedores $^{26,31}$ e os inibidores ${ }^{14,25}$ da absorção de Fe. Daí se deduz que a absorção de Fe na dieta pode ser aumentada, não só aumentando o seu conteúdo mas também aumentando a sua biodisponibilidade, o que se consegue aumentando a quantidade dos nutrientes que favorecem a absorção de $\mathrm{Fe}$ ou reduzindo a dos que a inibem. Portanto, conforme estabelecem Hallberg et al. ${ }^{115}$, existe uma grande influência potencial sobre os níveis de Fe em função da composição da dieta; de fato, na avaliação dos efeitos esperados por conta dessas modificações deve-se levar em conta o efeito da dieta de modo global do que de cada um dos alimentos em separado.

Alguns trabalhos demonstraram que a presença na dieta de determinados nutrientes relacionados com o metabolismo do Fe pode influir sobre a absorção, transporte e armazenamento do Fe no organismo ${ }^{115,116}$. Desta forma, nutrientes como as proteínas, proteínas de origem animal, Fe total, Fe hemo, cobre, vitamina $\mathrm{C}$, ácido fólico e vitamina $\mathrm{B}_{12}$, entre outros, são motivos de estudo quanto à sua relação com as reservas de $\mathrm{Fe}$ no organismo, avaliadas a partir dos níveis de ferritina sérica. Os resultados encontrados são muito díspares entre si, apresentando em alguns casos correlações muito fracas ${ }^{84,90}$, enquanto que em outros são descritas correlações significativas entre a ferritina sérica e as ingestas de $\mathrm{Fe}$ total ${ }^{81,117}$, proteínas de origem animal 109,118 e percentual de proteínas ${ }^{36,118}$. Como é um tema bastante atual, não será surpresa que em um futuro próximo haja novos achados e interpretações a respeito.

\section{2) A suplementação farmacológica de Fe:}

Antes de estabelecer qualquer tipo de terapia com ferro, deve-se ter uma boa justificativa, além de se descartar qualquer outra patologia orgânica oculta. Posteriormente, nos atletas que apresentem estados deficitários de Fe comprovados, após as condutas dietéticas adequadas deve-se recorrer à suplementação farmacológica de Fe. Surgem então duas questões muito importantes: Todos os atletas devem tomar suplementos de Fe preventivamente? Qual dose e qual tipo de Fe devem ser ingeridos?

Quanto à primeira questão, não existem evidências conclusivas de que a suplementação de $\mathrm{Fe}$ em atletas sem anemia manifesta traga algum efeito benéfico ${ }^{57}$. De fato, está demonstrado que nos atletas que apresentem problemas 
específicos de déficit de $\mathrm{Fe}$, as terapias de suplementação desse mineral podem melhorar nitidamente o rendimento físico ${ }^{119,120}$. Portanto, somente devem tomar suplementos os atletas nos quais se detectou previamente um déficit de $\mathrm{Fe}$, fundamentalmente através dos níveis de hemoglobina, ferritina sérica e percentual de saturação da transferrina. Nos atletas, e principalmente nos de alto nível, é recomendável o acompanhamento regular dos parâmetros hematológicos e em particular dos depósitos de $\mathrm{Fe}$ (ferritina sérica) ao longo da temporada, com o objetivo de avaliar eventuais reduções e estabelecer oportunamente as condutas adequadas de suplementação $0^{8,121}$.

Quanto à dose farmacológica adequada, dependerá da intensidade da deficiência de Fe. Existe uma ampla variação de critérios, com doses que podem oscilar desde os $60 \mathrm{mg}$ ao dia recomendados por Nickerson et al. ${ }^{122} \mathrm{em}$ corredoras de fundo até os $324 \mathrm{mg}$ ao dia, administrados por Erp-Baart et al. ${ }^{78}$ aos ciclistas participantes do Tour da França.

Tão importante quanto a quantidade de Fe é o tipo de preparado de Fe que será utilizado. Os sais de Fe farmacológicos possuem diferente solubilidade, biodisponibilidade e, em última análise, uma diferente absorção intestinal. Deve-se também enfatizar que o mais importante não é a quantidade total de sal de $\mathrm{Fe}$ do preparado, mas o equivalente real de $\mathrm{Fe}$ que este contém. A escolha do sal de $\mathrm{Fe}$ tem uma grande importância, não somente pelo seu melhor aproveitamento mas também pelas possíveis interferências que pode ocasionar sobre a absorção de outros minerais. Estudos recentes demonstraram que a suplementação com citrato férrico altera a absorção de magnésio, enquanto que o ascorbato ou o sulfato ferroso não só trazem uma boa absorção de Fe como também de cálcio e magné$\operatorname{sio}^{123}$.

A nossa experiência mostrou que são os sais de sulfato ferroso os que oferecem melhores resultados. Na tabela 5 , resumimos as condutas de suplementação de $\mathrm{Fe}$ seguidas no nosso Serviço para os atletas com ferropenia, a duração do tratamento e outras considerações gerais que devem ser levadas em conta.

$\mathrm{O}$ aporte de $\mathrm{Fe}$ farmacológico também tem os seus senões. Sabe-se que o $\mathrm{Fe}$ é o maior gerador de radicais livres e que estes, embora sejam necessários para a sobrevivência celular, são citotóxicos em grandes quantidades ${ }^{95}$. Por outro lado, demonstrou-se que um número importante de indivíduos (em torno de $20 \%$ da população americana) possuem um gene relacionado com o aumento da absorção intestinal de $\mathrm{Fe}^{124}$, razão pela qual nesses indivíduos além do correspondente aumento da produção de radicais livres será produzida uma sobrecarga desnecessária de Fe no organismo. Na Europa se estima que a prevalência de hemo- cromatose heterozigota seja ao redor de $10 \%$ e da forma hemozigota entre 0,3 e $0,5 \%$ da população.

Desta forma, é extremamente importante não utilizar a suplementação de Fe de forma indiscriminada. De fato, nos atletas com ferropenias francas, a suplementação de Fe está indicada, já que o Fe está intimamente ligado com o transporte de oxigênio e a instalação do quadro de anemia do atleta implica uma maior dependência do metabolismo anaeróbico, com as conseqüências que este fato acarreta. Por outro lado, a intenção de que se absorva uma pequena quantidade adicional de $\mathrm{Fe}$, que pode ser em torno de $1 \mathrm{mg}$ ao dia, contrasta com a quantidade total de Fe existente no organismo, a qual avaliamos anteriormente entre $3.000 \mathrm{e}$ $5.000 \mathrm{mg}$.

\section{TABELA 5}

Condutas de suplementação com ferro seguidas em nosso Serviço para atletas com ferropenia

Suplementação de acordo com o grau de deficiência de Fe:

1) Anemia manifesta (hemoglobina $<14 \mathrm{~g} \cdot \mathrm{dl}^{-1}$ no homem e $<12 \mathrm{~g} \cdot \mathrm{dl}^{-1}$ na mulher:

Dose: $160 \mathrm{mg}$ de Fe ao dia

em casos refratários, até $240 \mathrm{mg}$ de Fe ao dia

2) Ferropenias latentes ou pré-latentes (ferritina $<20$ ng. $\mathrm{ml}^{-1}$ ):

Dose: 160mg de Fe ao dia

3) Reduções dos níveis de ferritina ao longo da temporada (controles seriados):

Dose: dependem da velocidade com que reduzem os depósitos de Fe

\section{Duração do tratamento:}

- Pelo menos de dois a dois meses e meio.

- Após este período: novo controle laboratorial sem cessar a suplementação de Fe.

- Ajustar a dose de acordo com a resposta dos níveis de ferritina, hemoglobina e percentual de saturação da ferritina.

\section{Considerações gerais:}

- Ingerir o Fe pelo menos meia hora antes dos alimentos.

- Preferivelmente tomar Fe em forma de sais de sulfato ferroso.

- Se houver doenças digestivas, utilizar outro tipo de sais de Fe.

- Tomar junto com sucos de frutas cítricas (contêm vitamina C).

- Não se deve administrar o Fe indiscriminadamente sem indicação (prevenção), a menos que o atleta seja suscetível a uma redução em determinadas épocas da temporada (antecedentes prévios).

- Em anemias francas, recomenda-se reduzir a intensidade do treinamento, até que se tenha recuperado o estado normal hematológico. 
Outro tema de interesse atual é a suplementação conjunta de $\mathrm{Fe}$ e vitamina $\mathrm{C}$. Sabe-se que a vitamina $\mathrm{C}$ possui um poder redutor sobre o $\mathrm{Fe}$, favorecendo a sua passagem de forma férrica a ferrosa para uma melhor absorção intestinal, mas também se comprovou que um alto nível sérico de vitamina $\mathrm{C}$ reduzida facilita a sua penetração pelos poros da superfície da estrutura protéica da ferritina, convertendo o $\mathrm{Fe}^{+++}$em Fe $\mathrm{Fe}^{++}$catalítico, que se filtra através de tais poros para o exterior da molécula, gerando bilhões de radicais livres ${ }^{95}$. O papel de faca de dois gumes desempenhado pela vitamina $\mathrm{C}$, antioxidante por um lado e próoxidante por outro, a convertem em um importante tema de pesquisa na atualidade.

Em determinadas ocasiões, embora sejam seguidas corretamente as condutas de tratamento com Fe para os atletas anêmicos, observa-se uma fraca resposta quanto ao aumento da concentração de hemoglobina e especialmente os níveis de ferritina sérica. São os denominados casos refratários que Newhouse et al. ${ }^{83}$ creditam a uma má-absorção devida a interrelações do Fe com outros nutrientes ou devido a um problema genético.

Outro problema importante são as suplementações inadequadas de $\mathrm{Fe}$, aconselhadas em muitas ocasiões por in-

\section{REFERÊNCIAS}

1. Finch CA, Huebers H. Perspectives in iron metabolism. N Engl J Med 1982;306:1520-8.

2. De Mayer E, Adiels-Tegman M. The prevalence of anaemia in the world. World Health Stat Q 1985;38:302-16.

3. Hermosa V, Mazo E, Carril J, Cordovilla JJ, Luceño A, Zubizarreta A. Estudio prospectivo sobre la prevalencia de ferropenia en la población adulta de Cantabria. Med Clin 1986;87:135-40.

4. Viteri FE, Torun B. Anaemia and physical work capacity. Clin Haematol 1974;3:609-26.

5. Edgerton VR, Gardner GW, Ohira Y, Gunawardena KA, Senewiratne B. Iron deficiency anaemia and its effects on worker productivity and activity patterns. Br Med J 1979;2:1546-9.

6. Hallberg L. Iron nutrition and food iron fortification. Semin Hematol 1982;19:31-41.

7. Weaver CM, Rajaram S. Exercise and iron status. J Nutr 1992;122: 782 7.

8. Clement DB, Sawchuk LL. Iron status and sport performance. Sports Med 1984;1:65-74.

9. Swearingen JV. Iron deficiency in athletes: consequence or adaption in strenous activity. J Orthop Sports Phys Ther 1986;7:192-5.

10. Dallman PR. Manifestations of iron deficiency. Semin Hematol 1982; 19:19-30.

11. Pate RR. Sports anemia: a review of the current research literature. Phys Sports Med 1983;11:115-31.

12. Yoshimura H. Anemia during physical training (sports anemia). Nutr Rev 1970;10:251-3.

13. Dallman PR. Biochemical basis for the manifestations of iron deficiency. Ann Rev Nutr 1986;6:13-40. divíduos sem conhecimento e experiência no assunto, o que acaba por produzir diversos erros e um equilíbrio inadequado desse nutriente no organismo ${ }^{125}$. A suplementação de Fe, assim como o seu controle adequado e acompanhamento do seu metabolismo no atleta, deve ser competência exclusiva do médico.

Comparado com outros micronutrientes, são conhecidos muito mais aspectos do metabolismo do $\mathrm{Fe}$ do que de nenhum outro, embora persistam algumas interrogações. O conhecimento de tudo que foi exposto anteriormente parece de fundamental importância para o diagnóstico precoce e tratamento adequado das deficiências de $\mathrm{Fe}$, com especial ênfase no acompanhamento dietético dos atletas. Devese enfatizar que diagnosticar uma anemia do atleta equivale de certa forma a um fracasso, já que pode influenciar negativamente o desempenho de um atleta durante um grande período de tempo e na pior das hipóteses arruinar uma temporada previamente planejada.

\section{AGRADECIMENTOS}

Ao Dr. Enric Gimferrer da Unidade de Ferropatologia do Hospital de la Santa Creu i Sant Pau de Barcelona pela revisão e pelas sugestões feitas sobre o manuscrito original.

14. Linder MC. Nutrición, aspectos bioquímicos, metabólicos y clínicos Pamplona: Ed. Eunsa, 1988:189-241.

15. Vives JL. Anemia ferropénica y otros transtornos del metabolismo del hierro. In: Sans-Sabrafen J. Hematología clínica. Barcelona: Ed. Doyma, 1988:177-93.

16. Haymes EM. Nutritional concerns: need for iron. Med Sports Exerc 1987;5(Suppl):S197-S200.

17. Anderson L, Dible MV, Turkki PR, Mitchell HS, Rynbergen HJ. Nutrición y dietética de Cooper. Mexico, D.C.: Ed. Interamericana, 1988: 75110 .

18. Kutchai H. Gastrointestinal system. In: Berne RM, Levy MN. Principles of physiology. Missouri: Ed. Mosby-Year Book, 1996:437-501.

19. García JD, Crespo L, Guerra JM. Anemias ferropénicas. JANO 1990; 38:43-50

20. Haymes EM, Lamanca JJ. Iron loss in runners during exercise: implications and recommendations. Sports Med 1989;7:277-85.

21. National Research Council Committee of Dietary Allowances. Recommended dietary allowances. $10^{\text {th }}$ ed. Washington, D.C.: National Academy Press, 1989.

22. Hallberg L, Hogdahll AM, Nilsson L, Rybo R. Menstrual blood loss: a population study. Variation at different ages and attempts to define normality. Acta Obstet Gynecol Scand 1966;45:320-51.

23. Dallman PR. Changing iron needs from birth through adolescence. In: Fomon SJ, Zlotkin S, editors. Nutritional anemias. Nestlé Nutrition Workshop Series, Vol. 30. New York: Raven Press, 1992:29-38.

24. Watts E. Athletes' anaemia. A review of possible causes and guidelines on investigation. Br J Sports Med 1989;23:81-3.

25. Hallberg L. Bioavailability of dietary iron in man. Ann Rev Nutr 1981; 1:123-47. 
26. Monsen ER, Hallberg L, Layrisse M, Hegsted DM, Cook JD, Mertz W, et al. Estimation of available dietary iron. Am J Clin Nutr 1978;31: 13441.

27. Raper NR, Rosenthal JC, Woteki CE. Estimates of available iron in diets of individuals 1 year old and older in the Nationwide Food Consumption Survey. J Am Diet Assoc 1984;84:783-7.

28. McArdle WD, Katch FI, Kath VL. Exercise physiology. Philadelphia: Lea-Febiger, 1991.

29. Rasmussen EB, Hallberg L, Isaksson B, Arvidsson B. Food iron absorption in man. Applications of the two-pool extrinsic tag method to measure heme and nonheme iron absorption from the whole diet. J Clin Invest 1974;53:247-55.

30. Bauer JD. Hemoglobina, porfiria y metabolismo del hierro. In: Kaplan LA, Pesce AJ. Química clínica. Buenos Aires: Ed. Panamericana, 1988: 721-73.

31. Macphail P, Bothwell TH. The prevalence and causes of nutritional iron deficiency anemia. In: Fomon SJ, Zlotkin S, editors. Nutritional anemias. Nestlé Nutrition Workshop Series, Vol. 30. New York: Raven Press, 1992:1-12.

32. Newhouse LJ, Clement DB. Iron status in athletes. An update. Sports Med 1988;5:337-52.

33. Brotherhood J, Brozovic B, Pugh LGC. Haematological status of middle- and long-distance runners. Clin Sci Molec Med 1975;48:139-45.

34. Blum SM, Sherman AR, Boileau RA. The effects of fitness-type exercise on iron status in adult women. Am J Clin Nutr 1986;43:456-63.

35. Hunding A, Jordal R, Paulev PE. Runner's anemia and iron deficiency. Acta Med Scand 1981;209:315-8.

36. Telford RD, Cunningham RB, Deakin V, Kerr DA. Iron status and diet in athletes. Med Sci Sports Exerc 1993;25:796-800.

37. Clement DB, Asmundson RC. Nutritional intake and hematological parameters in endurance runners. Phys Sports Med 1982;10:37-43.

38. Moffat RJ. Dietary status of elite female high school gymnasts. Inadequacy of vitamin and mineral intake. J Am Diet Assoc 1984;84: 1361-3.

39. Lopez MA, Nuviala RJ, Abós D, Giner A. Estado nutricional del hierro en gimnastas de rítmica y nadadoras premenárquicas. Arch Med Dep 1989;6:47-55.

40. Snyder AC, Dvorak LL, Roepke JB. Influence of dietary iron source on measures of iron status among female runners. Med Sci Sports Exerc 1989;21:7-10.

41. Selby GB, Eichner ER. Endurance swimming, intravascular hemolysis, anemia, and iron depletion. Am J Med 1986;81:791-4.

42. Szygula Z. Erythrocytic system under the influence of physical exercise and training. Sports Med 1990;10:181-97.

43. Williamson MR. Anemia in runners and other athletes. Phys Sports Med 1981;9:73-9.

44. O'Neil F, Hynak-Hankinson MT, Gorman J. Research and application of current topics in sports nutrition. J Am Diet Assoc 1986;86:1007-15.

45. Nuviala RJ, Lapieza MG, Ansón JL, Castillo MC, Giner A. Efectos de una carrera de maratón sobre los parámetros hematológicos minerales y elementos traza. Arch Med Dep 1993;10:413-20.

46. Halvorsen FA, Lyng J, Ritland S. Gastrointestinal bleeding in marathon runners. Scand J Gastroenterol 1989;21:493-7.

47. Siegel AJ, Hennekens CH, Solomon HS, Boeckel BV. Exercise-related hematuria. Findings in a group of marathon runners. JAMA 1979;241: 391-2.

48. Seiler D, Nagel D, Franz H, Hellstern P, Leitzmann C, Jung K. Effects of long-distance running on iron metabolism and hematological parameters. Int J Sports Med 1989;10:357-62.
49. Weight LM, Byrne MJ, Jacobs P. Haemolytic effects of exercise. Clin Sci 1991;81:147-52.

50. McCabe ME, Peura DA, Kadakia SC, Bocek Z, Johnson LF. Gastrointestinal blood loss associated with running a marathon. Dig Dis Sci 1986; 31:1229-32.

51. McMahon LF, Ryan MJ, Larson D, Fisher RL: Occult gastrointestinal blood loss in marathon runners. Ann Intern Med 1984;100:846-7.

52. Katsumura T, Iwanf H, Shimomitsu T, Takanami Y, Ohya Y, Sakamoto A, et al. Change in serum iron after triathlon - an effect of small amount of iron. In: Hermans GPH, Mosterd WP, editors. Sports, medicine and health. Amsterdam: Excerpta Medica, 1990:487-91.

53. Vellar OD. Studies on sweat losses of nutrients. Scand J Clin Lab Invest 1968;21:157-67.

54. Parr RB, Bachman MA, Moss RA. Iron deficiency in female athletes. Phys Sports Med 1984;12:81-6.

55. Eichner ER. Sports anemia, iron supplements and blood doping. Med Sci Sports Exerc 1992;24:315-8.

56. Frederickson LA, Puhl JL, Runyan WS. Effects of training on indices of iron status of young female cross-country runners. Med Sci Sports Exerc 1983;15:271-6.

57. Weight LM, Noakes TD. Physical activity and iron metabolism. In: Bouchard C, Shephard RJ, Stepjens T, editors. Physical activity, fitness, and health. International Proceedings and consensus statement. Champaign: Humans Kinetics Publishers, 1994:456-70.

58. Finch CA, Bellotti V, Stray S, Lipschitz DA, Cook JD, Pippard MJ, et al. Plasma ferritin determination as a diagnostic tool. West J Med 1986; 145:657-63.

59. Walters GO, Miller FM, Worwood M. Serum ferritin concentration and iron stores in normal subjects. J Clin Path 1973;26:770-2.

60. Roberts D, Smith D. Serum ferritin values in elite speed and synchronized swimmers and speed skaters. J Lab Clin Med 1990;116:661-5.

61. Harju E, Pakarinen A, Larmi T. A comparison between serum ferritin concentration and the amount of bone marrow stainable iron. Scand J Clin Lab Invest 1984;44:555-6.

62. Wick M, Pinggera W, Lehmann P. Ferritin in iron metabolism. New York: Springer-Verlag Wien, 1991.

63. Jacobs A, Miller F, Worwood M, Beamish MR, Wardrop CA. Ferritin in the serum of normal subjects and patients with iron deficiency and iron overload. Br Med J 1972;4:206-8.

64. Gimferrer E. Fisiología e interés clínico del receptor de la transferrina. Bio-reguladores 1994;3:8-15.

65. Brittenham GM. New advances in iron metabolism, iron deficiency and iron overload. Curr Opin Hematol 1994;1:101-6.

66. Miller E, Brown B, Gorman D. The effects of hemoglobin supplements on maximal oxygen uptake in female athletes. J Manipulative Physiol Ther 1983;6:189-95.

67. Newhouse IJ, Clement DB, Tauton JE, McKenzie DC. The effects of prevalent/latent iron deficiency on physical work capacity. Med Sci Sports Exerc 1989;21:263-8.

68. Hercberg S. La carence en fer en nutrition humaine. Paris: Ed. Inter, 1988.

69. Buzina R, Grgic Z, Jusic M, Sapunar J, Milanovic V, Brubacher G. Nutritional status and physical working capacity. Hum Nutr Clin Nutr 1982;36:429-38.

70. Risser WL, Lee EJ, Poindexter HBW, West MS, Pivarnik JM, Risser $\mathrm{JMH}$, et al. Iron deficiency in female athletes: its prevalence and impact on performance. Med Sci Sports Exerc 1988;20:116-21. 
71. Reggiani E, Arras GB, Trabacca S, Senagera D, Chiodini G. Nutritional status and body composition of adolescent female gymnasts. J Sports Med 1989;29:285-8.

72. Nowak RK, Knudsen KS, Schulz LO. Body composition and nutrient intakes of college men and women basketball players. J Am Diet Assoc 1988;88:575-8

73. Brown RT, McIntosh SM, Seabolt VR, Danirl WA. Iron status of adolescent female athletes. J Adolesc Health Care 1985;6:347-52.

74. Diehl DN, Lohman TG, Smith SC, Kertzer RJ. The effects of physical training on the iron status of female athletes [abstr]. Med Sci Sports Exerc 1982;14:164.

75. Perron MS, Endres J. Knowledge, attitudes, and dietary practices of female athletes. J Am Diet Assoc 1985;85:573-6.

76. Pate RR, Sargent RG, Baldwin C, Burgess ML. Dietary intake of women runners. Int J Sports Med 1990;11:461-6.

77. Watkin VA, Myburgh KH, Noakes TD. Low nutrient intake does not cause the menstrual cycle interval disturbances seen in some ultramarathon runners. Clin J Sports Med 1991;1:154-61.

78. Erp-Baart AMJ, Saris WMH, Binkhorst RA, Vos JA, Elvers JWH. Nationwide survey on nutritional habits in elite athletes. Part II. Mineral and vitamin intake. Int J Sports Med 1989;10(Suppl a):S11-6.

79. Nuviala RJ, Castillo MC, Lapieza MG, Escanero JF. Iron nutritional status in female karatekas, handball and basketball players, and runners. Physiol Behav 1996;59:449-53.

80. Faber M, Benadé AJS. Mineral and vitamin intake in field athletes (discus-, hammer-, javelin-throwers and shotputters). Int J Sports Med 1991; 12:324-7.

81. Deuster PA, Kyle SB, Mase PB, Vigersky RA, Singh A, Schoomaker EB. Nutritional survey of highly trained women runners. Am J Clin Nutr 1986;45:954-62.

82. Lukaski HC, Hoverson BS, Gallargher SK, Bolonchuk WW. Physical training and copper, iron, and zinc status of swimmers. Am J Clin Nutr 1990;51:1093-9.

83. Newhouse IJ, Clement DB, Lai C. Effects of iron supplementation and discontinuation on serum copper, zinc, calcium, and magnesium levels in women. Med Sci Sports Exerc 1993;25:562-71.

84. Haymes EM, Spillman DM. Iron status of women distance runners, sprinters and control women. Int J Sports Med 1989;10:430-3.

85. Steinbaugh M. Nutritional needs of female athletes. Clin Sports Med 1984;3:649-70.

86. Food Nutrition Board. Recommended dietary allowances. $9^{\text {th }}$ ed. Washington D.C.: Ed. National Academy of Sciences, 1980.

87. Rowland TW, Kelleher JF. Iron deficiency in athletes. Insights from high school swimmers. AJDC 1989;143:197-200.

88. Lampe JW, Slavin JL, Apple FS. Poor iron status of women runners training for a marathon. Int J Sports Med 1986;7:111-4.

89. Nutter J. Seasonal changes in female athletes' diets. Int J Sports Nutr 1991;1:395-407.

90. Weight LM, Jacobs P, Noakes TD. Dietary iron deficiency and sports anaemia. Br J Nutr 1992;68:253-60.

91. Colt E, Heyman B. Low ferritin levels in runners. J Sports Med 1984;24: 13-7.

92. Durstine JL, Pate RR, Sparling PB, Wilson GE, Senn MD, Bartoli WP. Lipid, lipoprotein and iron status of elite women distance runners. Int J Sports Med 1987;8(Suppl 2):119-23.

93. Nickerson HJ, Tripp AD. Iron deficiency in adolescent cross-country runners. Phys Sports Med 1983;11:60-6.
94. Weight LM, Kleij M, Noakes TD, Jacobs P. 'Sports Anemia' - A real or apparent phenomenon in endurance-trained athletes? J Sports Med 1992;13:344-7

95. Herbert V, Shaw S, Jayatilleke E. Vitamin C-driven free radical generation from iron. J Nutr 1996;126:1213S-20S.

96. Sigal LH. The acute phase response to inflammation. In: Sigal LH, Ron Y, editors. Immunology and inflammation. Basic mechanisms and clinical consequences. New York: McGraw-Hill, Inc., 1994:287-301.

97. Tyrer LB. Nutritional and the pill. J Reprod Med 1984;29(Suppl): 547-50.

98. Balaban EP, Cox JV, Snell P, Vaughan RH, Frenkel EP. The frequency of anemia and iron deficiency in the runners. Med Sci Sports Exerc 1988;21:643-8.

99. Douglas PD. Effect of a season of competition and training on hematological status of women field hockey and soccer players. J Sports Med Phys Fitness 1989;29:179-83.

100. Pellicia A, Di Nucci GB. Anemia in swimmers: Fact or fiction? Study of hematologic and iron status in male and female top-level swimmers. Int J Sports Med 1987;8:227-23.

101. Meredith CN, Dwyer JT. Nutrition and exercise: Effects on adolescent health. Annu Rev Public Health 1991;12:309-33.

102. Rowland TW. Deficiencia de hierro en el deportista joven. Clin Ped North Am (ed. española) 1990;37:1213-24.

103. Smith NJ. Nutrition and the young athlete. Pediatr Ann 1978;7:682-9.

104. Burman D. Iron metabolism in infancy and childhood. In: Jacobs A, Worwood M, editors. Iron in biochemistry and medicine. New York: Academic Press, 1974:543-62.

105. De Wijn JF, Pikaar NA. Haemoglobin, serum iron and iron binding capacity during growth from age 9 to 17 . Semi-longitudinal study of boys and girls. Maandschr Kindergencesk 1970;38:29-36.

106. Hermosa J, Mazo E, Bureo E, Carril JJ, Cordovilla J, Zubizarreta A. Estudio prospectivo sobre la prevalencia de ferropenia en Cantabria entre niños de seis y catorce años. An Esp Pediatr 1987;27:275-80.

107. Fernández-Ballart J, Domenech-Massons JM, Salas J, Arija V, MartiHenneberg C. The influence of nutrient intake on the biochemical parameters of iron status in a healthy pediatric Mediterranean population. Eur J Clin Nutr 1992;46:143-9.

108. Moreiras-Varela O, Carbajal A, Blasquez MJ, Cabrera L, Martínez A. La alimentación en la escuela y en el hogar de niños madrileños: Estudio piloto. Rev Esp Pediatr 1984;40:257-66.

109. Salas J, Galan P, Arija V, Martí-Henneberg C, Hercberg S. Iron status and food intakes in a representative sample of children and adolescents living in a Mediterranean city of Spain. Nutr Res 1990;10:379-90.

110. Rowland TW, Stagg L, Kelleher JF. Iron deficiency in adolescent girls. Are athletes at increased risk? J Adolesc Health 1991;12:22-5.

111. Nuviala RJ, López-Benedicto MA, Abos D, Giner A. Iron status of female adolescent-swimmers and rhythmic gymnasts. In: Tsopanakis A, Poortmans J, editors. Physiological biochemistry of exercise and training. Athens: Hellenic Sports Research Institute 1986:297.

112. Romero I, Le fer: Perspectives chez des enfants et des adolescents avec activité physique. Mémoire de Recherche Scientifique. Université de Nancy (France), 1994.

113. Nuviala RJ, Lapieza MG, Castillo MC, Aspiroz MT, Abos D, Giner A. Efectos del entrenamiento físico sobre los parámetros hematológicos en jóvenes nadadores. Sangre 1992;37:363-7.

114. Mataix J, Mañas M, Llópis J, Martínez de Victoria E. Tablas de composición de alimentos españoles. Instituto de Nutrición y Tecnología de Alimentos. Universidad de Granada, 1993. 
115. Hallberg L, Rossander-Hultén L, Brune M. Prevention of iron deficiency by diet. In: Fomon SJ, Zlotkin S, editors. Nutritional anemias. Nestlé Nutrition Workshop Series, Vol 30, New York: Raven Press, 1992: 169-81.

116. Keith RE. Vitamins and physical activity. In: Wolinsky Y, Hickson JF Jr, editors. Nutrition in exercise and sport. Boca Rabon (USA): CRC Press, 1991:159-83.

117. Lamanca JJ, Haymes EM. Effects of low ferritin concentration on endurance performance. Int J Sport Nutr 1992;2:376-85.

118. Nuviala RJ, Lapieza MG. Disparity between diet and serum ferritin in elite sportswomen. Nutr Res 1997;17:451-61.

119. Clarkson PM. Minerals: exercise performance supplementation in athletes. J Sports Sci 1991;9:91-116.

120. Fogelholm M, Jaakkola L, Lampisjärvi T. Effects of iron supplementation in female athletes with low serum ferritin concentration. Int J Sports Med 1992;13:158-62.
121. Nuviala RJ, Elosegui LM, Puzo J, Abos D, Giner A. Cambios hematológicos y de minerales en ciclistas a lo largo de la temporada deportiva. Apunts Med l'Esport 1990;27:127-35.

122. Nickerson HJ, Holubets MC, Weiler BR, Haas RG, Schwartz S, Ellefson ME. Causes of iron deficiency in adolescent athletes. J Pediatr 1989; 114:657-63.

Traduzido por:

José Kawazoe Lazzoli

Editor-Chefe da Revista Brasileira de Medicina do Esporte

Vice-Presidente da Sociedade de Medicina Desportiva do Rio de Janeiro

Professor do Depto. de Morfologia e da Disciplina de Medicina do Exercício e do

Esporte, da Universidade Federal Fluminense, Niterói, RJ

Diretor do ERGOCENTER/Cor Diagnose, Petrópolis, RJ 